\title{
Gestión del conocimiento en las universidades
}

\section{Comunicar en entornos digitales}

\section{Knowledge management in universities}

\section{Communication in digital environments}

\section{Gestão do conhecimento nas universidades}

\section{Comunicação em ambientes digitais}

DOI: https://doi.org/10.18861/ic.2020.15.1.2953

\section{VIENA MEDINA GONZÁLEZ}

medina.viena@gmail.com - Universidad de Málaga, España.

ORCID: https://orcid.org/0000-0003-3568-7490

ANA ALMANSA MARTÍNEZ

anaalmansa@uma.es - Universidad de Málaga, España.

ORCID: http://orcid.org/0000-0003-0256-6369

\section{ANTONIO CASTILLO ESPARCIA}

acastilloe@uma.es - Universidad de Málaga, España.

ORCID: https://orcid.org/0000-0002-9751-8628
Fecha de recepción: 1 de febrero de 2020

Fecha de aceptación: 28 de abril de 2020

\section{RESUMEN}

El objetivo del trabajo es constatar la relación entre la comunicación y la gestión del conocimiento en el ámbito universitario, así como la comunicación del conocimiento a través del uso de plataformas digitales. La metodología empleada se encaminó a la descripción del objeto de estudio mediante la revisión teórica y documental, lo que permitió identificar a los principales conceptos, perspectivas de análisis y modelos asociados. Los resultados obtenidos pusieron foco en los elementos convergentes entrelas variables dela investigación, lo que permitió resaltar la relevancia de difundir el conocimientogenerado en la institución Universidad para apoyareldesarrollotanto organizacional como social.Como partedelasconclusiones, sedestacala conexión intrínseca de los procesos comunicativos con los elementos de la 
gestión del conocimiento. En tal sentido, las tecnologías juegan un papel esencial como herramientas para lograr un efectivo flujo de información en una universidad y obliga, en el contexto actual, a gestionar sus procesos en espacios digitales.

PALABRASCLAVE: gestión del conocimiento, comunicación del conocimiento, Universidad, plataformas digitales.

\section{ABSTRACT}

The objective of the work is to verify the relationship between communication and knowledge management in the university environment, as well as the communication of knowledgethrough the use of digital platforms. The methodology used was aimed at describing the object of study through a theoretical and documentary review, which allowed identifying the main concepts, analysis perspectives and associated models. The results obtained focused on the converging elements between

42 the variables of research, which allowed us to highlight the relevance of spreading the knowledge generated in the Univeristy institution to supportboth organizational and social development. As part of the conclusions, the intrinsic connection of communication processes with the elements of knowledge management is highlighted. In this sense, technologies play an essential role as tools to achieve an effective flow of information in a university and forces, in the current context, to manage its processes in digital spaces.

KEYWORDS: knowledge management, knowledge communication, University, digital platforms.

\section{RESUMO}

O objetivo do trabalhoéverificar a relação entre comunicação e gestão do conhecimento no ambiente universitário, bem como a comunicação do conhecimento através do uso de plataformas digitais. A metodologia utilizada focou na descrição do objeto de estudo por meio da revisão teórica e documental, que permitiu identificar os principais conceitos, perspectivas de análise e modelos associados. Os resultadosobtidosfocalizaramoselementos convergentes entre as variáveis da pesquisa, o que permitiu destacar a relevância da difusão do conhecimento gerado na instituição Universidade para apoiar o desenvolvimento organizacional e social. Como parte das conclusões, destaca-se a conexão intrínseca dos processos de comunicação aos elementos da gestão do conhecimento. Nessesentido, astecnologiasdesempenham umpapelessencial como ferramenta para alcançar um fluxo efetivo de informações em uma universidade e obriga, no contextoatual, agerenciarseus processosem espaçosdigitais.

PALAVRAS-CHAVE: gestão do conhecimento, comunicação do conhecimento, Universidade, plataformas digitais. 


\section{INTRODUCCIÓN}

La comunicación, como proceso en el que se comparten y transmiten experiencias, valores, ideas, opiniones, además de favorecer el intercambio entre individuos y entidades, es un pilar fundamental de la organización de la sociedad de la información y del conocimiento, al ser esta quien establece la utilidad verdadera de los activos mencionados (López, 2006). La comunicación propicia la creación y compartición del conocimiento, considerado hoy día un activo intangible imprescindible para el progreso de cualquier organización; de ahí que su identificación, adquisición, desarrollo, retención, difusión y utilización se consideren procesos estratégicos (León, 2008).

La Universidad, como espacio esencial de producción de conocimiento, se caracteriza por ser una institución orientada al cumplimento de una responsabilidad social específica, principalmente, por la necesidad de que los planes y las modalidades de estudio sean coherentes con lo que necesita y exige la sociedad para la cual se preparan los futuros profesionales. De ahí que lo señalado por Martín (2009) sobre que el primer desafío de la comunicación en la sociedad del conocimiento es poner a dialogar a la Universidad con su sociedad, deba ser una premisa fundamental en las políticas de comunicación de dicha institución.

La necesidad no solo de crear sino de compartir el conocimiento generado en las universidades, como entidades fundamentales en este accionar y transformadoras de procesos sociales, económicos, políticos y culturales, se ha convertido actualmente en una realidad imperiosa. Al respecto, es esencial fomentar ambientes de aprendizaje donde profesores, investigadores y estudiantes se encuentren en constante intercambio de conocimientos y que estos tributen al desarrollo investigativo, la calidad de la docencia, la sostenibilidad de proyectos institucionales, además de la creación de alianzas nacionales e internacionales.

$\mathrm{Al}$ respecto, este artículo se orienta a reflexionar sobre la relación entre la gestión del conocimiento (GC) y la comunicación en la Universidad a partir de la relevancia de la difusión del contenido de las tareas por dicha institución a través de sus plataformas digitales (sitios web, redes sociales, portal de comunicación científica, revistas digitales, Intranet, sitios de campañas promocionales o recaudación de fondos, repositorios). Este abordaje implica establecer como objetivo esencial la descripción de aspectos teóricos sobre comunicación, comunicación del conocimiento, GCy plataformas digitales en las universidades, así como la relación entre esos conceptos. 


\section{COMUNICACIÓN DEL CONOCIMIENTO EN LAS UNIVERSIDADES POR MEDIO DE PLATAFORMAS DIGITALES}

En la actual sociedad de la información y del conocimiento se ha vuelto esencial el uso de las tecnologías de la información y las comunicaciones (TIC) para una rápida y eficiente gestión de estos recursos. Todo ello acompañado de un proceso comunicativo orientado a facilitar el intercambio y la colaboración entre diversos actores sociales. En esa línea, la comunicación es una dimensión importante en el proceso de difusión y GC, y lo que refiere a las tecnologías, el liderazgo y la cultura (Özhan \& Özlem, 2009).

Para lograr que la GC se desarrolle de manera eficiente en los diversos espacios y, específicamente, en el contexto universitario, la comunicación se convierte en un eslabón esencial para facilitar el desarrollo del conocimiento en el ambiente interno y externo mediante su intercambio entre los diversos actores, lo cual permite, a su vez, la proyección de la institución y la puesta en práctica de mecanismos de aprendizaje y colaboración.

En el accionar delas instituciones universitarias se constata el fuerte vínculo entre la gestión de su conocimiento y la necesidad de mecanismos y estrategias que posibiliten la comunicación del conocimiento generado en la Universidad, lo cual abarca diferentes propósitos, aunque su finalidad tiene un interés social que está dado por la responsabilidad de esta institución.

\subsection{Comunicación organizacional y Comunicación del conocimiento}

Actualmente, es inevitable separar los conceptos de información, comunicación y conocimiento si se toma en cuenta el auge del desarrollo de las TIC, en cuyo marco surgen cada día nuevos espacios y dispositivos, y los usuarios se convierten en actores relevantes de la de la generación e intercambio de información, principalmente en los contextos digitales. Esta situación ha promovido cambios en los modelos de desarrollo de los países, afianzados en la necesidad de la informatización de la sociedad. En tal sentido, el modelo de sociedad dela información, dondela GCyla comunicación se vuelven esenciales, toma relevancia como proceso de carácter social que influye en los cambios de los individuos, las organizaciones y las sociedades. En este sentido, CastilloEsparcia y Smolak-Lozano (2013) señalan:

La plasmación de las redes sociales en la planificación estratégica de la comunicación en las organizaciones ha obligado a una profunda transformación de los procesos de gestión de la comunicación, de los mecanismos de actuación y de la evaluación de las campañas (p. 473).

Rizo García (2014) aborda la comunicación como un fenómeno sociocultural que va desde la comunicación interpersonal a la comunicación colectiva, 
pasando por sus múltiples modalidades (comunicación directa, comunicación mediada, comunicación digital) y por sus diversas áreas de aplicación (organizacional, política, educativa, para el desarrollo), además de tener en cuenta los nuevos fenómenos comunicativos: redes sociales, comunicación digital, hipermediaciones. De manera general, se define como un proceso en el que las personas manifiestan su capacidad de comunicar y que ha sido conceptualizado históricamente a partir de perspectivas diversas (Alonso \& Saladrigas, 2006): transmisión de información (paradigma informacional), intercambio de información y hacer común, compartir experiencias.

Siguiendo el criterio de Alonso y Saladrigas, la conceptualización del término puede verse desde dos enfoques privilegiados: transmisión (difusivo), en el que se define la comunicación por términos como emitir, difundir o dar información a otros; y el enfoque ritual (expresivo), donde es asociada a términos como participación, asociación y posesión de creencias y valores compartidos.

\subsubsection{Comunicación en las organizaciones}

Actualmente, la comunicación en las organizaciones se ha convertido en un proceso estratégico que influye en el logro delos objetivos y metas institucionales a partir de la eficiencia con que se utilizan los flujos y canales de información. A través de ellos los distintos niveles de la organización compartirán el conocimiento de las actividades y acciones que se realizan, además de optimizar los procesos y fomentar las relaciones inter y extraorganizacionales.

En las perspectivas presentadas se destacan elementos que identifican este proceso de manera general, como es el caso de la relevancia de las relaciones entrelos individuos de la organización por medio de los flujos comunicacionales. Igualmente se destaca la comunicación como proceso esencial para el cumplimiento de los objetivos organizacionales, en tanto habilita el intercambio de información, conocimiento, experiencias, convirtiéndose en un eslabón clave para lograr la compartición el conocimiento y, de esa manera, el desarrollo del capital intelectual. De esta manera se logra una identidad cultural y colectiva que caracteriza el clima organizacional y propicia la imagen de la organización más allá de sus límites internos. 
Tabla 1. Definiciones de comunicación organizacional

\begin{tabular}{|c|c|}
\hline Autor / Referencia & Definiciones \\
\hline Goldhaber (1984) & $\begin{array}{l}\text { Es el flujo de comunicaciones internas (ascendentes y descendentes y } \\
\text { horizontales) que se dan dentro de una organización, para establecer relaciones } \\
\text { entre los individuos que conforman la organización. }\end{array}$ \\
\hline Timm (1986) & $\begin{array}{l}\text { La comunicación es esencial para la organización, en el sentido de obtener } \\
\text { cooperación y consenso entre todos los integrantes de la empresa. }\end{array}$ \\
\hline Scheinsohn (1993) & $\begin{array}{l}\text { Hecho fundante de las relaciones, las cuales se entablan, mantienen y fomentan } \\
\text { a través de ella. Este autor considera que el acto comunicativo, cuando se } \\
\text { desarrolla entre sujetos sociales más complejos (públicos, instituciones, } \\
\text { gobiernos, reparticiones), agudiza sus complicaciones. }\end{array}$ \\
\hline Rojas y Arape (1999) & $\begin{array}{l}\text { El intercambio progresivo de informaciones, sentimientos o saberes destinados } \\
\text { al conocimiento y las transformaciones que logran el crecimiento común } \\
\text { de todas las partes involucradas; es decir, la comunicación es una fuerza } \\
\text { determinante en los procesos empresariales de transformación y no un } \\
\text { instrumento auxiliar para el desarrollo de estrategias. }\end{array}$ \\
\hline Vargas (2003) & $\begin{array}{l}\text { La condición de anunciar y conseguir técnicas de comunicación que permitan } \\
\text { incrementar verdaderamente el concepto de público o de audiencia que no } \\
\text { sólo reciba información, sino que además genere visiones y conductas que } \\
\text { favorezcan a la organización en el cumplimiento de sus objetivos. }\end{array}$ \\
\hline Lehtonen (2009) & $\begin{array}{l}\text { Interacción social a través de la cual se produce el intercambio y la creación de } \\
\text { conocimiento. }\end{array}$ \\
\hline $\begin{array}{l}\text { Fernández y Vásquez } \\
\text { (2011) }\end{array}$ & $\begin{array}{l}\text { La principal herramienta integradora a través de la cual se gestiona y garantiza } \\
\text { el éxito y la diferenciación de una organización frente a otras. }\end{array}$ \\
\hline Saladrigas (2018) & $\begin{array}{l}\text { Proceso sociocultural complejo de producción, transmisión, intercambio e } \\
\text { interpretación de formas simbólicas por los integrantes de una organización } \\
\text { con identidad colectiva, a partir de los recursos materiales y culturales con } \\
\text { los que cuentan y las posiciones que ocupan en sus contextos históricamente } \\
\text { específicos e institucionalmente estructurados. }\end{array}$ \\
\hline
\end{tabular}

Fuente: Elaboración propia.

La comunicación y la cultura organizacional, al estar fuertemente relacionadas en la compartición de significados y patrones de comportamiento que caracterizan a la entidad, dan lugar a que la comunicación se convierta en una responsabilidad compartida ligada a la información que se maneja y a los puestos de trabajo (Guzmán, 2012). De ahí que el desarrollo de las habilidades comunicativas se convierta en una prioridad organizacional.

Fontalvo, Quejada y Puello (2011) puntualizan que una comunicación organizacional efectiva establece medios para informar a las partes interesadas sobre los objetivos y los procesos requeridos para satisfacer las necesidades del cliente, así como de su seguimiento, evaluación y mejora en función de las labores que realiza y su posición en la organización. Siguiendo el criterio de los autores, esto igualmente se relaciona con el desarrollo de las competencias de las personas, la estructura y el funcionamiento de los procesos y el conocimiento generado a partir de la relación entre las partes de la organización con su ambiente externo. 


\subsubsection{Comunicación del conocimiento}

Con respecto a las diversas perspectivas de análisis, se considera pertinente destacar la de Castells (1997), quien, a partir de su concepción de la información como proceso, permite comprender la dinámica comunicativa como un todo y entender la comunicación del conocimiento. De esta manera se explicita la triada información-comunicación-conocimiento como activos intangibles dependientes y pueden definir a una sociedad a partir de los modelos de cambio en los que juegan un rol esencial, principalmente en un contexto de digitalización.

Meza $(2013 ; 2015)$ entiende a la comunicación del conocimiento como el diálogo que se produce en la práctica discursiva científica para hacer circular el conocimiento. Eppler (2004) acota que comunicar conocimiento es más que comunicar información, ya que depende de los espacios de intercambio, las experiencias, las percepciones y los valores de las personas, además de tener en cuenta la cultura de la organización y el desarrollo de ambientes de confianza y normas de colaboración para garantizar una efectiva transferencia de conocimiento en todos los niveles.

En lo que respecta a la comunicación del conocimiento profesional en el plano gerencial, Straub y Karahanna (1998) la consideran una actividad clave pensar la especialización laboral actual. Según los autores, la transferencia eficiente y efectiva de experiencias y conocimientos entre diferentes expertos y los responsables de la toma de decisiones es un requisito previo para la coordinación de procesos de calidad y las acciones organizacionales. En tal sentido, Liyanage, Ballal, Elhag \& Li (2009) se basan en la teoría de la comunicación para crear su modelo de transferencia de conocimiento y poder explicar el acto de colaboración entre la fuente y el receptor, además de tener en cuenta el modelo de conversión de conocimiento de Nonaka y Takeuchi (1995).

La comunicación del conocimiento, como concepto, se incluye en la explicación del subproceso de compartición/distribución del conocimiento (Probst, Raub \& Romhardt, 2001) como parte del proceso estratégico de GC en el que, indiscutiblemente, la comunicación es un eslabón esencial para poder llevar a cabo este tipo de gestión en las organizaciones. Algo que León (2008) afirma cuando expone su propuesta de acciones dentro de cada subproceso de GC y, el caso de la compartición, señala lo imprescindible de:

- Perfeccionar el proceso de comunicación.

- Determinar el conocimiento que puede ser difundido.

- Analizar la potencialidad del conocimiento.

- Determinar emisor- receptor.

- Determinar canales de difusión.

- Entregar conocimiento. 


\subsection{Gestión del conocimiento}

En la década de los 90 del pasado siglo, el conocimiento comenzó a adquirir un gran valor para aquellas entidades que necesitaban cambios en su quehacer diario y establecer ventajas competitivas ante las nuevas realidades tecnológicas, políticas y económicas. Entornos cambiantes que demandaban el desarrollo de nuevas competencias profesionales y donde las necesidades del mercado exigían una mayor competitividad. De ahí el surgimiento y el desarrollo de prácticas orientadas a la obtención de la calidad de los procesos, productos y servicios, basadas en el aprendizaje organizacional.

Según León (2008), la interrelación del conocimiento, su gestión y el aprendizaje llevan a las organizaciones hacia nuevos enfoques y procesos, así como a la innovación y al cambio. La autora señala que la capacidad de generar procesos dinámicos de aprendizaje constituye el elemento más crítico en todoslos niveles y conduce a un dominio formado por las relaciones entre el conocimiento, el sujeto que conoce y el entorno sobre el cual ese sujeto actúa con base en ese conocimiento. En el caso de las entidades, la necesidad de crear y trasmitir conocimiento se concreta en el proceso de GC, el cual se orienta a la identificación, adquisición, uso, desarrollo y difusión de este activo intangible. A continuación, se presentan consideraciones de diversos autores sobre el tema.

Tabla 2. Definiciones de gestión del conocimiento

\begin{tabular}{|c|c|}
\hline Autor / Referencia & Definiciones \\
\hline Van Buren (2000) & $\begin{array}{l}\text { Consiste en adquirir, utilizar y mejorar los conocimientos necesarios para la } \\
\text { organización, creando un ambiente que permita compartirlos y transferirlos } \\
\text { entre los trabajadores para que los utilicen en vez de volver a descubrirlos. }\end{array}$ \\
\hline Wiig (2002) & $\begin{array}{l}\text { La GC se relaciona con el comportamiento humano, competencias, } \\
\text { habilidades, actitudes, filosofías empresariales, modelos, operaciones, } \\
\text { prácticas y tecnologías. A su vez puede considerarse como una filosofía, } \\
\text { estrategia, proceso, objetivo, sistema o práctica organizacional. }\end{array}$ \\
\hline $\begin{array}{l}\text { Davenport y Prusak } \\
(2001)\end{array}$ & $\begin{array}{l}\text { Consiste en usar el conocimiento en la práctica. También consiste en lograr } \\
\text { objetivos institucionales mediante la capacitación de personas, estructuración } \\
\text { de tecnologías y contenidos; la creación de depósitos de conocimiento, la } \\
\text { mejora del acceso al conocimiento y el perfeccionamiento de las culturas y los } \\
\text { ámbitos de conocimiento. }\end{array}$ \\
\hline $\begin{array}{l}\text { Gupta, Lakshmi y } \\
\text { Aronson }(2000)\end{array}$ & $\begin{array}{l}\text { Proceso que ayuda a la organización a encontrar, diseminar y transferir } \\
\text { importante conocimiento tácito y explícito, y la experticia necesaria para } \\
\text { actividades como: resolver problemas, tomar decisiones, desarrollar } \\
\text { planificación estratégica o aprendizaje dinámico. }\end{array}$ \\
\hline $\begin{array}{l}\text { Järvenpää y Mäki } \\
(2001)\end{array}$ & $\begin{array}{l}\text { Incluye creación de nuevo conocimiento, conocimiento compartido y difusión, } \\
\text { y métodos para promover estas acciones. }\end{array}$ \\
\hline $\begin{array}{l}\text { Molina y Marsal } \\
(2002)\end{array}$ & $\begin{array}{l}\text { La GC implica formular, formalizar, rehusar y contribuir al incremento del } \\
\text { conocimiento de la estructura social. }\end{array}$ \\
\hline $\begin{array}{l}\text { Passoni y Zanfrillo } \\
(2003)\end{array}$ & $\begin{array}{l}\text { La GC está esencialmente basada en una buena gestión de la información. } \\
\text { Proceso sistemático basado en la capacidad de seleccionar, organizar, presentar } \\
\text { y usar la información por parte de los miembros de la organización, con el } \\
\text { objetivo de utilizar en forma cooperativa los recursos de conocimiento basados } \\
\text { en el capital intelectual propio, y con la finalidad de desarrollar las aptitudes } \\
\text { organizacionales y la generación de valor. }\end{array}$ \\
\hline
\end{tabular}




\begin{tabular}{|c|c|}
\hline Ponjuán (2006) & $\begin{array}{l}\text { Proceso sistemático e integrador de coordinación de las actividades de } \\
\text { adquisición, creación, almacenaje y comunicación del conocimiento tácito y } \\
\text { explícito por individuos y grupos con objeto de ser más efectivos y productivos } \\
\text { en su trabajo, y así cumplir los objetivos y metas de la organización. }\end{array}$ \\
\hline Pérez-Montoro (2008) & $\begin{array}{l}\text { Disciplina que se encarga del estudio del diseño y de la implementación } \\
\text { de sistemas cuyo principal objetivo es que todo el conocimiento tácito, } \\
\text { explicito, individual, interno y externo involucrado en la organización } \\
\text { pueda transformarse y convertirse, sistemáticamente, en conocimiento } \\
\text { organizacional o corporativo. }\end{array}$ \\
\hline Hoffmann (2009) & $\begin{array}{l}\text { La GC comprende un conjunto de procedimientos establecidos para } \\
\text { desenvolver y controlar todo tipo de conocimiento tácito y explícito para que la } \\
\text { organización atienda sus objetivos. }\end{array}$ \\
\hline $\begin{array}{l}\text { García y Valentim } \\
(2014)\end{array}$ & $\begin{array}{l}\text { La GC consiste en una teoría y práctica que busca promover una cultura } \\
\text { positiva en relación a la generación, socialización, apropiación y comunicación } \\
\text { del conocimiento en un determinado ambiente, a partir de la aplicación de } \\
\text { métodos y técnicas específicas que habiliten generar un conjunto de acciones } \\
\text { que a su vez permitan valorizar el conocimiento de la organización en beneficio } \\
\text { de sí misma o del ambiente que le rodea. }\end{array}$ \\
\hline
\end{tabular}

Fuente: Elaboración propia.

La mayoría de los estudios abordados se basan en las personas como capital esencial en las organizaciones, teniendo en cuenta sus conocimientos y competencias. Y establecen cómo la GC potencia este capital para lograr transformaciones organizacionales orientadas al acceso, la comunicación, la cultura organizacional, el uso de la información, además de la creación y compartición del conocimiento en pos de la toma de decisiones, la solución de problemas y la obtención de beneficios.

La GC como proceso encargado de desarrollar el conocimiento dentro de la organización y hacia el exterior, posibilita la creación de nuevo capital intelectual, y con ello habilita el progreso basado en el uso de este activo. De manera general, favorece el funcionamiento de las entidades, permitiendo un mejor uso y aprovechamiento del conocimiento existente en favor del cumplimiento de las estrategias y objetivos organizacionales, la obtención de productos y servicios eficientes, además de la creación de espacios donde el conocimiento individual y estructural se considera el activo primordial.

\subsection{Comunicación y la gestión del conocimiento en las organizaciones}

Cuando se hace referencia a los procesos de comunicación y GC es imposible separar las acciones a las que están asociadas, ya que durante la conversión del conocimiento una depende de la otras (Nonaka \& Takeuchi, 1995), a lo cual se suma la necesidad de los mecanismos de comunicación de lograr un adecuado intercambio y compartición del conocimiento generado en la organización que es necesario transmitir a todos los niveles y a su ambiente externo.

Treem (2012) acota que para crear conocimiento es extremadamente importante la actitud con la que se comunica la experiencia y la forma en que 
se comparte. En la investigación realizada por Grigorescu, Lupu y Al Zink (2014) sobre la comunicación como vector central en la GC (Huete, 2018), se presentan aspectos dentro dela comunicación yla GC que pueden considerarse esenciales para las actividades organizacionales, entre los que se mencionan: cultura organizacional abierta al intercambio de ideas, interacción con asociaciones, preservación de la memoria organizacional, compartición de conocimiento, estrategias de comunicación, dinámicas de socialización, prestación de servicios de información, existencia de bases de datos, comunicación con otras organizaciones (redes de cooperación), comunicación mediante las tecnologías y los recursos humanos.

De igual forma, estos autores señalan como clave las redes de cooperación para el uso colectivo del conocimiento generado; de esta manera se procesa información y se desarrollan instrumentos de comunicación que pueden apoyar al aprendizaje, la investigación y la innovación. Al respecto se acota que la proyección, inserción y gestión de programas de asociación con otras organizaciones son acciones necesarias para estimular la cooperación científica y valorar el potencial de las TIC en las estrategias y actividades utilizadas en la cooperación. De esta forma se ayuda también a incrementar la calidad de los productos y los servicios que se ofrecen.

Sobre los procesos comunicativos y los flujos de información y su importancia para la distribución del conocimiento en las organizaciones (Alavi \& Leidner, 1999), algunos autores (Gupta \& Govindarajan, 1996; Szulanski, 1996) establecen que la relación entre los elementos del proceso comunicativo y los que forman parte de la distribución del conocimiento es fundamental. Y destacan algunos de los siguientes elementos: el valor del conocimiento de la fuente; la disposición motivacional de la fuente; la existencia y efectividad de los canales de transmisión; la disposición motivacional del receptor para adquirir conocimiento de la fuente; la capacidad de adquirir, asimilar y usar el conocimiento por parte del receptor; las características del mensaje (tipo de conocimiento); y el contexto en el que se maneja la comunicación y la relación entre la fuente y el receptor.

De manerageneral, para lograr el desarrollo de redes de colaboración y conformación de grupos de trabajo con el objetivo de potenciar el conocimiento organizacional, se hacen necesarios los mecanismos de comunicación, tanto formales como informales, unidos a las normas de colaboración y coordinación entre los miembros del equipo y a nivel organizacional.

Özhan y Özlem (2009) afirman que la comunicación, al igual que las tecnologías, el liderazgo y la cultura, es una importante dimensión de la GC, utilizada principalmente para compartir el conocimiento explícito. Al respecto, se destacan las relaciones que se establecen entre los individuos que permiten comunicar dicho conocimiento, principalmente en la etapa de creación y compartición, y las TIC, que desempeñan un papel importante (Mohr, 2007). 
Con el objetivo de representar el rol de la comunicación en las prácticas de la GC, Özhan y Özlem (2009) presentan un modelo basado, a su vez, en el modelo SECI de Nonaka y Takeuchi (1995), en el que se explicitan las maneras de comunicar -verbal, escrita o digital- según las fases de conversión del conocimiento durante su flujo en el contexto organizacional.

Diagrama 1. El modelo de importancia de la comunicación en la creación y el intercambio de conocimiento

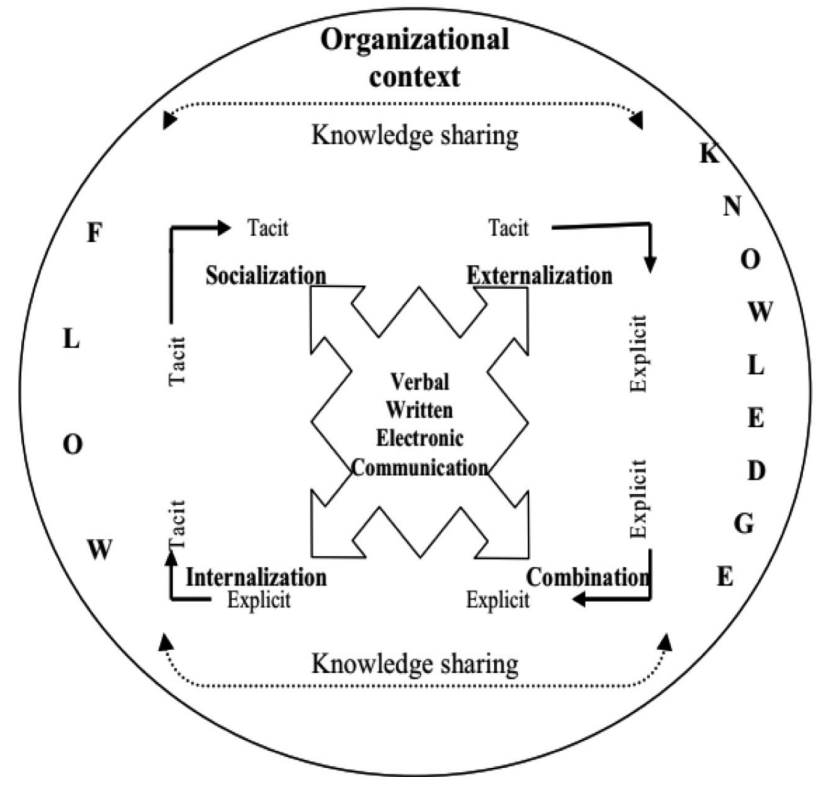

Fuente: Özhan y Özlem (2009).

El modelo confirma que la organización necesita desarrollar constantementeherramientas comunicativas para lograr el efectivo flujo de conocimiento. Un estudio realizado (Crawford \& Strohkirch, 2006) demuestra que a medida que las personas tienen más competencias comunicativas favorecen la GCy son más capaces de realizar las acciones asociadas al proceso; principalmente adquirir, crear y compartir.

Con respecto al tema, Fontalvo, Quejada y Puello (2011) consideran esencial señalar que la comunicación al interior de las empresas puede acelerar, detener o anular los procesos de innovación y de generación de conocimiento, ya que, según los autores, de la eficacia de los mecanismos de comunicación dependen el entendimiento de las políticas y objetivos de la organización, el establecimiento de rutinas eficientes de trabajos, el sistema de documentación, el flujo dinámico de información entre los niveles y el mejoramiento del aprendizaje. 
En este marco, la GCy los procesos comunicacionales son la base de las actividades innovadoras, al permitir a la entidad generar nuevos conocimientos, difundirlos entre sus empleados y materializarlos en productos o en mejoras organizacionales (Fontalvo, Quejada \& Puello, 2011).

\section{METODOLOGÍA}

Se realizó una investigación de tipo descriptiva y se empleó la técnica de revisión teórica y documental. Las principales fuentes de información revisadas fueron artículos de revistas, capítulos de libros, tesis doctorales, ponencias en eventos y páginas web.

La revisión permitió el estudio de los aspectos teóricos relacionados con la comunicación del conocimiento, el proceso de comunicación, la GC, el uso de plataformas digitales y, por último, su relación en el contexto universitario. En esta línea, se consiguió la identificación de perspectivas de análisis, los principales autores y los modelos asociados.

A partir de los resultados obtenidos se abrió una discusión crítica de los modelos teóricos y metodológicos.

\section{RESULTADOS}

\subsection{Comunicación y gestión del conocimiento en las universidades}

Los contextos universitarios son considerados verdaderos espacios de creación y transmisión de conocimiento como parte del cumplimiento de su responsabilidad social: formar futuros profesionales capaces, a partir del conocimiento y las competencias adquiridas, de transformar y desarrollar las diversas esferas de la sociedad en las que son insertados una vez que culminan sus estudios. Por lo tanto, su modelo comunicativo debe adecuarse a las exigencias comunicativas de las sociedades, pasando de un enfoque unidireccional a uno circular y dialógico, en cuyo marco las acciones comunicativas que desplieguen constituyan vías de interconexión entre los colectivos internos y externos (Almansa-Martínez \& Alonso García, 2012).

Leite (2007) considera a las actividades de generación de conocimiento como propias de espacios universitarios, y cada organización será capaz, según sus características, de aplicar la GC. Este proceso es visto en universidades como forma de incentivar la socialización y la comunicación de conocimiento (García \& Valentim, 2014). 
Por otra parte, Galbreath (2000) puntualiza que, desdela perspectiva del conocimiento académico, la comunidad de aprendizaje debe comenzar desde el nivel individual, crear conocimiento a nivel de departamentos y crear dominios de conocimiento alrededor de los departamentos que permitan compartir los intereses académicos o disciplinares, además de crear redes de conocimiento institucional y con otras entidades.

De manera general, se considera que para la creación de estrategias de GC que impliquen la comunicación como una de sus dimensiones principales, se puede tener cuenta el criterio de Estrada y Benítez (2006), quienes destacan la creación de comunidades de conocimiento por ramas afines del saber e interdisciplinarias que garanticen la relación humana necesaria para - a su vezgarantizar un flujo eficaz de conocimientos. También se necesita disponer de herramientas que permitan el intercambio de información, conocimientos y experiencias; mecanismos de actualización sistemática de la información; disponer de bases de datos que beneficien a estudiantes, profesores, investigadores y expertos; gestionar el conocimiento tácito y convertirlo en conocimiento explícito; y disponer de un repositorio de información de conocimiento al que tengan acceso los diferentes públicos.

El contexto universitario exige una gestión comunicacional que incluya a la GC y el apoyo a la investigación, la innovación y el desarrollo del capital intelectual de la institución. La misma permite tomar decisiones acordes a lo que exige la sociedad y garantizar la transparencia de la información y el conocimiento que se genera, desarrolla y difunde. De ahí la necesidad de contar con una política de comunicación institucional y su conexión transversal con las estrategias de GC.

\subsubsection{Modelos de GC relacionados con la comunicación en el contexto universitario}

Los modelos que se abordan a continuación confirman que la puesta en práctica dela GC en las universidades facilita la transferencia del conocimiento allí generado en favor del desarrollo organizacional y en favor de sociedad en sentido amplio. Todo esto está unido a las facilidades que ofrecen las tecnologías para transferir el conocimiento como parte del proceso comunicativo.

A continuación, se describen de manera sucinta aquellos modelos cuyos elementos son de interés para la presente investigación. 
Tabla 3. Modelos de gestión del conocimiento

\begin{tabular}{|c|c|}
\hline Modelo & Enfoques \\
\hline $\begin{array}{l}\text { Modelo de gestión } \\
\text { del conocimiento } \\
\text { (Passoni \& } \\
\text { Zanfrillo, 2003). }\end{array}$ & $\begin{array}{l}\text { Orientado a departamentos académicos, tiene por objetivo insertar el modelo en la gestión de unidades } \\
\text { académicas en instituciones de educación superior. Se basa en etapas para su creación, que a su vez se } \\
\text { relacionan con los procesos de la GC: } 1 \text {. identificación, recolección y selección; } 2 \text {. descubrimiento del } \\
\text { conocimiento; 3. compartir 4. aplicar. } \\
\text { Las autoras concluyen su estudio enfatizando la relevancia de los modelos de GC en estos contextos, } \\
\text { esencialmente para actividades de Evaluación Institucional y Acreditación de Carreras, ya que permiten } \\
\text { tomar decisiones y elaborar estrategias a partir del conocimiento oportuno. Se puntualiza que las } \\
\text { personas representan un factor clave para el éxito de dicha gestión. }\end{array}$ \\
\hline $\begin{array}{l}\text { Modelo de gestión } \\
\text { de conocimiento } \\
\text { científico (Leite, } \\
\text { 2006). }\end{array}$ & $\begin{array}{l}\text { Considera que las actividades de GC en ambientes académicos se presentan a partir de procesos de } \\
\text { comunicación, y en este marco no debe solamente considerarse las tecnologías, sino principalmente } \\
\text { la influencia y la responsabilidad de la comunicación en la compartición de conocimiento, el } \\
\text { aprendizaje y la cultura organizacional. Este autor propone un modelo denominado Gestión de } \\
\text { Conocimiento Científico orientado al contexto académico, donde considera los siguientes factores: } \\
\text { ambiente académico, comunidades académicas, comunicación científica, cultura y procesos de mapeo, } \\
\text { adquisición, organización y almacenamiento, compartición y creación de conocimiento. }\end{array}$ \\
\hline $\begin{array}{l}\text { Modelo de gestión } \\
\text { del conocimiento } \\
\text { (Correa, Rosero } \\
\text { \& Segura, 2008). }\end{array}$ & $\begin{array}{l}\text { Está dirigido a la Escuela Interamericana de Bibliotecología (EIB) en Colombia. Dicho modelo se basa } \\
\text { en el modelo Intelect (Euroforum, 1998) y se orienta hacia una concepción estratégica que, según los } \\
\text { autores, representa la globalidad de la GC y expresa lo que se debe hacer para contribuir a la innovación } \\
\text { como base para lograr la competitividad en la EIB: memoria organizacional, red de canales de } \\
\text { conocimiento, generación de conocimiento e innovación. } \\
\text { También se tienen en cuenta las áreas estratégicas de la universidad para la creación del modelo: } \\
\text { Desarrollo de la ciencia, la tecnología y la innovación; Formación humanística y científica de excelencia; } \\
\text { Interacción Universidad-sociedad; Bienestar universitario y Gestión universitaria. } \\
\text { Según los autores, el modelo permitirá identificar y estructurar en forma relacionada: Los } \\
\text { conocimientos de las personas, sus capacidades, destrezas y talento / El reconocimiento de la } \\
\text { Universidad / La comunidad académica / La sociedad / La calidad e intensidad de las relaciones que se } \\
\text { mantienen con personas e instituciones del ambiente educativo / Los conocimientos propios de la EIB } \\
\text { para realizar sus procesos administrativos frente a la docencia, la investigación y la extensión. }\end{array}$ \\
\hline $\begin{array}{l}\text { Modelo de gestión } \\
\text { del conocimiento } \\
\text { (Pérez, Ramírez } \\
\text { \& Gallego, 2011). }\end{array}$ & $\begin{array}{l}\text { Creado para el Instituto Tecnológico Metropolitano de Medellín (Colombia), sus autores lo definen } \\
\text { como una propuesta para gestionar la creación de conocimiento, estableciendo una interacción } \\
\text { dinámica entre el conocimiento tácito y el conocimiento explícito. Para ello, las personas que forman } \\
\text { parte de la organización deben trabajar en función de generar, conservar y transmitir el conocimiento, y } \\
\text { crear una nueva cultura y una dinámica organizacional que provea su mejora continua. } \\
\text { El modelo establece un conjunto de elementos básicos a tener en cuenta: Alineación con la misión, } \\
\text { visión estratégica y filosofía institucional / Las personas como el ente esencial en la GC / El capital } \\
\text { intelectual como valor primordial de la institución / El valor de uso del conocimiento / Tecnología / } \\
\text { Liderazgo. } \\
\text { Resulta interesante el carácter sistémico que se le atribuye a esta propuesta por tener en cuenta todas } \\
\text { las partes del sistema y sus procesos. Se puntualizan aspectos como: el acceso al conocimiento desde } \\
\text { todos los niveles, la transmisión y adquisición del conocimiento por medio de redes y el intercambio de } \\
\text { información interna y externa, dando lugar a un aprendizaje continuo. } \\
\text { Como elementos constitutivos del modelo se proponen: Necesidades del entorno en relación a la } \\
\text { institución / Conocimiento organizacional: Relacionado con los procesos de la organización y aspectos } \\
\text { referentes a la cultura y clima organizacional, la capacidad organizacional y tecnológica, las políticas y } \\
\text { estrategias / Red de GC: Las personas y organizaciones establecen vínculos para generar, transmitir y } \\
\text { compartir conocimiento. Se destacan las comunidades de práctica. }\end{array}$ \\
\hline $\begin{array}{l}\text { Modelo } \\
\text { Hexagonal } \\
\text { de gestión del } \\
\text { conocimiento } \\
\text { (Lopera \& } \\
\text { Quiroz, 2013). }\end{array}$ & $\begin{array}{l}\text { Modelo aplicable a las funciones universitarias de investigación y extensión, en cuyo marco se } \\
\text { consideran dos niveles para la propuesta de componentes, un nivel estratégico orientado a la definición } \\
\text { de la estrategia, los procesos, la estructura y la medición; y un nivel operativo que contempla las acciones } \\
\text { de las personas, la información y la tecnología. } \\
\text { Dicho modelo tiene por objetivo articular los procesos de investigación y extensión, proponer una } \\
\text { estrategia organizacional orientada a la GC, propiciar la mejora continua de los procesos basados en } \\
\text { el conocimiento, evaluar las acciones de GC y sus resultados, identificar y transmitir las fuentes de } \\
\text { conocimiento eficazmente, fomentar la innovación, favorecer el acceso a la información y apoyar la } \\
\text { toma de decisiones. }\end{array}$ \\
\hline
\end{tabular}




\begin{tabular}{|c|c|}
\hline $\begin{array}{l}\text { Modelo de gestión } \\
\text { de conocimiento } \\
\text { científico (García } \\
\text { \& Valentim, } \\
\text { 2014). }\end{array}$ & $\begin{array}{l}\text { Orientado a un ambiente universitario, concretamente para el área de Ciencia de la Información de la } \\
\text { Universidad Estadual Paulista (Brasil). Según las autoras, el modelo persigue el objetivo de presentar } \\
\text { algunas especificidades del conocimiento generado en esa área. Se ha basado en los procesos que } \\
\text { componen la dinámica del conocimiento científico según Valentim (2005): asimilación, generación, } \\
\text { socialización y comunicación /diseminación. } \\
\text { El modelo se divide en cuatros ambientes y un núcleo. Los primeros representan los contextos que } \\
\text { deben tenerse en cuenta para la GC y lo segundo representa la dinámica de conocimiento científico: } \\
\text { 1. Ambiente externo: Entidades externas a la universidad; } 2 \text {. Ambiente organizacional: Relaciona } \\
\text { elementos pertinentes del contexto de la universidad y características organizacionales; } 3 \text {. Ambiente } \\
\text { departamental: Relaciona elementos estructurales del contexto interno de la universidad; } 4 \text {. Ambiente } \\
\text { de comunidad académico-científica: Directamente relacionado con la elaboración de conocimiento } \\
\text { científico. }\end{array}$ \\
\hline $\begin{array}{l}\text { Factores Clave } \\
\text { de Exito en la } \\
\text { Implantación } \\
\text { de Sistemas } \\
\text { de Gestión del } \\
\text { Conocimiento } \\
\text { (SGC) en } \\
\text { Instituciones } \\
\text { de Educación } \\
\text { Superior (De } \\
\text { Freitas \& Yaber, } \\
\text { 2015). }\end{array}$ & $\begin{array}{l}\text { Propuesta agrupada en tres factores (humanos, gerenciales y tecnológicos) e influenciados, según } \\
\text { los autores, por otros elementos que forman parte de la GC, por lo que debe enfocarse de manera } \\
\text { sistemática y considerar los aspectos internos y externos de la institución: la cultura organizacional, } \\
\text { el enfoque de la calidad total (personas, procesos, servicios, productos), el plan estratégico de la } \\
\text { universidad en conjunto con la estrategia de GC, de gestión de información y de comunicación, la } \\
\text { vigilancia tecnológica y los aspectos de evaluación. } \\
\text { En el caso de los aspectos externos se hace referencia a aquellos aspectos económicos, políticos, } \\
\text { sociales, medioambientales y tecnológicos sobre los cuales la institución no toma decisiones y pueden } \\
\text { convertirse en amenazas u oportunidades. }\end{array}$ \\
\hline
\end{tabular}

Fuente: Elaboración propia.

El estudio de los modelos anteriores indica que existen convergencias en la fundamentación de sus elementos identificativos, lo cual facilita determinar los componentes de la GC en el espacio universitario. Al respecto se destacan las etapas de creación, desarrollo y socialización, y las personas como factor clave; además de otros elementos como la cultura organizacional, el liderazgo, las tecnologías y la comunicación entre las áreas de trabajo. Todo ello concatenado con los espacios de intercambio y la existencia de redes de información y conocimiento entre los individuos y organizaciones que tributan a las acciones de aprendizaje.

Igualmente se identifican diferencias entre las propuestas, esencialmente en sus objetivos y, según el contexto de estudio, los elementos que consideran significativos como parte de la GC: toma de decisiones (Passoni \& Zanfrillo, 2003), procesos de comunicación (Leite, 2006), planificación estratégica basada en competitividad e innovación (Correa, Rosero \& Segura, 2008), la dinámica de la interacción del conocimiento (Pérez, Ramírez \& Gallego, 2011; García \& Valentim, 2014), articulación de procesos organizacionales (Lopera y Quiroz, 2013) y la GC desde un enfoque sistémico y articulado con otros procesos (Pérez, Ramírez \& Gallego, 2011; De Freitas \& Yaber, 2015).

De igual forma se aprecia que los modelos estructuran la GC por niveles (estratégicos y operativos) o ambientes (organizacional o externo), donde se destacan los espacios de creación de conocimiento, articulación entre las personas y toma de decisiones; así como las relaciones que facilitan el intercambio con el entorno. Esto igualmente depende delas características dela institución, 
las demandas sociales en cuanto al conocimiento científico necesario para la solución de problemas y el grado en que se considera la GC un proceso estratégico y transversal.

\subsection{Las TIC y plataformas digitales como medios para comunicar el conocimiento en las universidades}

Las TIC son consideradas herramientas de apoyo a la GC en tanto contribuyen a que el conocimiento de una organización, o el adquirido en fuentes externas, pueda ser compartido por diversas vías y de manera rápida favoreciendo los procesos productivos, investigativos y de desarrollo. Rodríguez y otros autores (2009) señalan que el rol o función que cumplen las TIC es facilitar la conservación y el almacenamiento del conocimiento, su organización y categorización, así como brindar las posibilidades para compartirlo, pero sobre todo acelerar la velocidad de transferencia de dicho conocimiento.

Becerra y Leidner (2014) destacan los repositorios como sistemas de la GC que ayudan a capturar y reutilizar dicho activo y en los que se demuestra el favorable rol de la tecnología en su gestión. No obstante, las TIC solamente constituyen el medio y el sistema de almacenamiento para el intercambio del conocimiento. No generan conocimiento y no pueden garantizar, ni siquiera promover, la generación del conocimiento o su intercambio en una cultura institucional que no favorezca esas actividades (Davenport \& Prusak, 2001a).

Unido a este rol de las TIC en la GC se encuentra el uso extensivo de herramientas comunicativas (Martín, 2011; La Roda, 2016; Castillo-Esparcia, 2008) a partir del desarrollo de un modelo de comunicación interactiva basado en los cibermedios (Cebrián, 2009), en cuyo marco la selección adecuada de una plataforma digital permite desarrollar procesos de comunicación del conocimiento a escala global. Esto último permite además que especialistas e investigadores, con diversos niveles de experiencia, pongan al día su saber, orientados hacia la práctica y el diálogo (Pérez \& Castañeda, 2009).

Referente al tema se destaca la comunicación digital como herramienta efectiva para difundir los contenidos institucionales, y su gestión se considera un elemento imprescindible para la identidad y la consolidación de las organizaciones (Puertas, Cadme \& Álvarez, 2015; Álvarez, 2011).

Al respecto, las TICy, en específico, la comunicación digital, son utilizadas en las universidades como herramientas que apoyan el aprendizajeyla creación de redes de conocimiento donde alumnos, profesores einvestigadores desarrollan el autoaprendizaje mediante el dominio de los navegadores y buscadores de información: Es decir, a través de diversos mecanismos de interactividad con diversas redes sociales o de conocimiento y los enlaces e interrelaciones con las fuentes de información tanto internas como externas.

En este caso se encuentran los entornos virtuales de enseñanza-aprendizaje, donde las TIC se convierten en tecnologías para el aprendizaje y el conoci- 
miento colaborativo(Dorado, 2006). "Los resultados alertan sobrela necesidad de fomentar, tanto a nivel individual como institucional, ecologías de aprendizaje más enriquecidas, para que cada docente pueda aprovechar mejor las posibilidades de aprendizaje que ofrece la sociedad en red" (González-Sanmamed, Estévez, Souto-Seijo \& Muñoz-Carril, 2020, p. 9).

También se destacan los sistemas de GC que permiten organizar y almacenar el conocimiento generado en la Universidad (Munir \& Rohendi, 2012).Con el objetivo de conectar a las universidades con la realidad social y así obtener resultados de impacto y colaboración, surgen en dicho contexto los medialabs. Romero y Robinson (2017) los consideran como un tipo de laboratorio centrado en la experimentación con tecnologías y medios de comunicación, encaminados a convertirse en laboratorios de mediación ciudadana e innovación educativa, social y digital donde ciudadanos e investigadores comparten conocimientos. Los autores señalan que estos laboratorios muestran un enfoque de: ciudadanía activa, creatividad, experimentación, flexibilidad, innovación social, transferencia de conocimiento (Universidad/sociedad y sociedad/ Universidad), actitud emprendedora y activismo en favor del conocimiento abierto. Igualmente acotan como relevante la utilización de las redes sociales como herramientas de monitoreo y medición del impacto social de los resultados alcanzados.

Como parte de las herramientas de comunicación en universidades, se destacan las sedes webs (Almansa-Martínez \& Alonso García, 2012). Carrillo, Castillo y Blanco (2016) la consideran "el eje central de la comunicación en la vida universitaria y de los servicios de información, abarcando la docencia, la investigación y la gestión" (s.p.). Estos autores señalan que "los principales objetivos de las sedes webs universitarias son: publicar y diseminar actividades y resultados de investigación, proporcionar información administrativa y académica de interés para los estudiantes, y facilitar la colaboración con otras instituciones nacionales e internacionales" (s.p.); convirtiéndose en un medio estratégico parala difusión de conocimiento einformación, además dela transmisión de la marca institucional.

En lo que respecta a las redes sociales, estas pueden funcionar igualmente como espacios facilitadores de conocimiento en la relación estudiante-estudiante, estudiante-profesor y Universidad-sociedad. "La web 2.0 permite que la organización interactúe con sus públicos y con los usuarios" (Reina, Fernández \& Noguer, 2012, p. 123), comunicándose sin limitaciones temporales o espaciales. Da Costa (2004) señala que estas redes son un factor clave para comprender la lógica de la acción colectiva y su evolución. La utilización de la web 2.0 "permite a las universidades tener estilos de formación más activos con feedback en los procesos de aprendizaje, en la construcción y difusión del conocimiento, en la creación, diseño, elaboración y publicación de información en línea" (Carrillo, Castillo \& Blanco, 2016, s.p.). 
De manera general, las plataformas digitales han producido cambios significativos en la educación al generar nuevas formas de transferencia del conocimiento (Barrera \& Guapi, 2018). Las organizaciones han podido aprovechar los beneficios de los espacios digitales para difundir el conocimiento que generan, además de transmitir y compartir la información derivada de los procesos institucionales y de sus relaciones con otras organizaciones.

Cadena y Melgarejo (2017) señalan que las plataformas digitales facilitan la comunicación, colaboración y la eficiencia, y tienen como objetivo impulsar la colaboración entre los principales usuarios (investigadores de las universidades, industria y gobierno) llevándolos a incrementar el impacto de la investigación. Estos autores hacen alusión a los tipos de plataformas digitales utilizadas en las universidades (académica, comercial, red social y fuente). Igualmente las describen como:

- Plataformas digitales para comunicación externa: usadas por los investigadores para comunicar o coordinarse con pares externos. Enfoque colaborativo.

- Plataformas digitales para productividad: usadas por los investigadores para administrar sus proyectos de investigación. Enfoque académico/comercial/redes sociales.

- Plataformas digitales para interacción: usadas por los investigadores para comunicarse con su equipo de investigación (investigadores y estudiantes). Enfoque académico/comercial/redes sociales.

- Plataformas digitales usadas para difusión de la investigación: repositorios, Open Access Repositorios Journals, blogs, bases de datos. Enfoque de publicación.

\section{CONCLUSIONES}

El conocimiento se ha convertido en el activo intangible más importante en eldesarrollo delas organizaciones. Por esa razón el proceso dela GC se considera esencial para la toma de decisiones, ya que se encuentra estrechamente relacionado con la planificación estratégica y los procesos organizacionales. Cuestión que necesita de un proceso comunicacional que facilite la compartición /distribución del conocimiento y que permita asílograr un aprendizaje organizacional quefavorezcala potenciación del capital intelectualy una cultura organizacional basada en el compromiso yla motivación. Esto igualmente propiciaría eficientes flujos de información y canales de comunicación que generen alianzas y lazos de colaboración entre los actores involucrados en los procesos organizacionales y a nivel de la sociedad. 
Las universidades como actores clave de la vida social generan conocimiento relevante y deben generar condiciones que promuevan su intercambio y socialización. Por lo tanto, sus resultados de investigación e innovación necesitan de procesos comunicacionales capaces de difundir sus acciones con el objetivo de transformar y beneficiar a la sociedad, ya sea a partir de los propios procesos académicos, investigativos o los proyectos de extensión universitarios.

Actualmente, es esencial el uso y la explotación de las plataformas digitales como soporte para la divulgación y la promoción de los proyectos institucionales y de desarrollo en los que se involucra la universidad. Al respecto, las TIC son consideradas un componente de apoyo de la GC junto a la comunicación. De esta manera la institución da a conocer el conocimiento que genera, los resultados de trabajo alcanzados, las relaciones establecidas con colaboradores y rinde cuentas sobre el cumplimiento de su responsabilidad social (Durat \& Mengual, 2014).

Los modelos de GC presentados, a pesar de diferenciarse en cuanto a objetivos y estructuras, mantienen un enfoque hacia los factores identificativos de la GC, los cuales son señalados de manera indistinta, pero se resumen en lo siguiente: las personas, sus conocimientos y competencias; los espacios de interacción y socialización de prácticas y experiencias; las políticas yestrategias de GC; la cultura organizacional; el liderazgo; las tecnologías; las acciones de medición; y los recursos de apoyo. De manera general, orientan sobre cómo estructurar el proceso según el tipo de contexto y alcance (nivel estratégico: planificación estratégica; y nivel operativo: creación, desarrollo y socialización del conocimiento). Además de tener en cuenta los ambientes tanto organizacionales como externos.

\section{REFERENCIAS}

Alavi, M. \& Leidner, D. E (1999). Knowledge Management and Knowledge Management System: Conceptual Foundation and Research Issues. Paris: INSEAD Working Paper Series.

Almansa-Martínez, A. \& Alonso García, M.M. (2012). Innovación en las salas de prensa virtuales de las universidades andaluzas. En E. Ordeix \& J. A. Rom Rodríguez (coord.), La innovació en relacions publiques (pp. 429-440). Barcelona: AIRP.

Alonso, M. \&Saladrigas Medina, H.(2006). Teoría de la comunicación. Una introducción a su estudio. La Habana: Pablo de la Torriente.

Álvarez, A. (2011). Medición y Evaluación en Comunicación. Málaga: Instituto de Investigación en Relaciones Públicas. 
Barrera Rea, V. F. \& Guapi Mullo, A. (2018). La importancia del uso de las plataformas virtuales en la educación superior. Revista Atlante: Cuadernos de Educación y Desarrollo. Recuperado de: https://www.eumed.net/rev/atlante/2018/07/ plataformas-virtuales-educacion.

Becerra Fernández, I. \& Leidner, D. (2014). On knowledge, knowledge management, and knowledge management system. An Introduction. En I. Becerra Fernández \& D. Leidner (Eds.), Knowledge management. An evolutionary view (pp. 3-10). Armonk, New York: M.E. Sharpe.

Cadena Vinueza, P. \& Melgarejo Heredia, R. (2017). Impacto del uso de plataformas digitales para la investigación en el área de administración de empresas en las universidades ecuatorianas. Simposio Argentino sobre Tecnología y SociedadJAIIO 46, 61-71.

Carrillo Durán, M. V., Castillo Díaz, A. \& Blanco Sánchez, T. (2016). La comunicación en las sedes webs de las universidades a distancia españolas. Málaga: Asociación Española de Investigación de la Comunicación.

Castells, M. (1997). La era de la información. Madrid: Ed. Alianza.

Castillo-Esparcia, A. (2008): Estudio sobre el uso de las tecnologías de la comunicación en la comunicación interna, Anagramas, 12(6), 93-106.

Castillo-Esparcia, A. \& Lozano, E (2013): Redes sociales y organizaciones. Modelos de evaluación, Historia y Comunicación Social, 18, 473-487. DOI: https://doi. org/10.5209/rev_HICS.2013.v18.44343

Cebrián Herreros, M. (2009). Nuevas formas de comunicación: cibermedios y medios móviles. Comunicar, XVII 33, 10-13.

Correa Uribe, G., Rosero Jiménez, S.L. \&SeguraJiménez,H.(2008).Diseñodeun modelo de gestión del conocimiento para la Escuela Interamericana de Bibliotecología. Revista Interamericana de Bibliotecología, 31(1), 85-108.

Crawford, C. B. \& Strohkirch, C. S. (2006). The Critical Role of Communication in Knowledge Organizations: Communication Apprehension as a Predictor of Knowledge Management Functions. Journal of Knowledge Management Practice, $7(4)$, (s.p.).

Da Costa, R. (2004). Inteligencia afluente e açao coletiva. A expansao des redes sociais e problema da assimetria individuo/grupo. Razón y palabra, 41, (s.p.).

Davenport, T. \& Prusak, L. (2001). Conocimiento en Acción. Cómo las organizaciones manejan lo que saben. Buenos Aires: Pearson Education.

Davenport, T. \& Prusak, L. (2001a). ¿De qué hablamos cuando hablamos del conocimiento? En T. Davenport \& L. Prusak. Conocimiento en Acción. Cómo las organizaciones manejan lo que saben. Buenos Aires: Pearson Education. 
De Freitas, V. \& Yaber, G. (2015). Una Taxonomía de los Factores Clave de Éxito en la Implantación de Sistemas de Gestión del Conocimiento en Instituciones de Educación Superior GECONTEC. Revista Internacional de Gestión del Conocimiento y la Tecnología, 3(1), 69-86.

Dorado Perea, C. (2006). El trabajo en red como fuente de aprendizaje: posibilidades y límites para la creación de conocimiento. Una visión crítica. Educar, 37, 11-24.

Durat, J. M. \& Mengual Andrés, S. (2014). Impacto de la Sociedad del Conocimiento en la Universidad y en la comunicación científica. RELIEVE Revista Electrónica de Investigación y Evaluación Educativa, 20(2), 1-12.

Eppler, M. J. (2004). Knowledge Communication Problems between Experts and Managers. An Analysis of Knowledge Transfer in Decision Processes. Paper 1, Institute for Corporate Communication, Faculty of Communication Sciences, University of Lugano

Estrada, V. \& Benítez, F. (2006). La Gestión de conocimiento en la nueva universidad cubana. En AA.VV. La nueva universidad cubana y su contribución a la universalización del conocimiento, 49-58. La Habana: Félix Varela.

Euroforum. (1998). Modelo de medición del Capital Intelectual. Madrid: Euroforum Escorial.

Fernández, A. \& Vázquez, M. (2011). Creación de un Gabinete de Comunicación y establecimiento de un plan de comunicación-base para la Eurorregión GaliciaNorte de Portugal. Revista Razón y Palabra, 74. Recuperado de: http://www. razonypalabra.org.mx

Fontalvo Herrera, T. J., Quejada, R. \& Puello Payares, J. G. (2011). La gestión del conocimiento y los procesos de mejoramiento. Dimens. Empres, 9(1), 80-87.

García, C. L. \& Valentim, M. L. (2014). Gestão do Conhecimento Científico: Proposta de um modelo para a área de Ciência da Informação da Universidade Estadual Paulista (UNESP). Tendências da Pesquisa Brasileira em Ciência da Informação, 7(1), (s.p.).

Galbreath, J. (2000). Knowledge management technology in education: An overview. Educational Technology, 40(5), 28-33.

Goldhaber, G. (1984). Comunicación Organizacional. México: Diana.

González-Sanmamed, M., Estévez, I., Souto-Seijo, A. \& Muñoz-Carril, P.C. (2020). Ecologías digitales de aprendizaje y desarrollo profesional del docente universitario. Comunicar, XXVIII(62), 9-18.

Grigorescu, A., Lupu, M. M. \& Al Zink, F. (2014). Communication - Central Vector in Knowledge. Management Strategies Journal, 26(4), 538-548. 
Gupta, A. \& Govindarajan, V. (1996). Determinants of knowledge outflows from and inflows into foreign subsidiaries in multinational corporations. Working Paper, Robert H. Smith School of Business, University of Maryland at College Park, MD.

Gupta, B., Lakshmi, L.S. \& Aronson, J.E. (2000). Knowledge management: practices and challenges. Industrial management and data systems, 100(1), 17-21.

Guzmán Paz, V. (2012). Comunicación organizacional. México: Red Tercer Milenio S.C.

Hoffmann, W. A. M. (2009). Gestão do conhecimento: desafíos de aprender. São Carlos: Compacta.

HueteFlores,E.J.(2018).Lacomunicación comoestrategiadedesarrolloyorganizacional en la gestión del conocimiento. Revista Economía y Administración, 9(2), (s.p.).

Järvenpää, E. \& Mäki, E. (2001): Knowledge sharing in networked organizations. Boston: Butterworth Heinemann.

La Roda Gómez, A. (2016). La comunicación interna de la calidad en la universidad española. Propuesta de un modelo teórico trasmedia. Tesis de Doctorado. Departamento de Comunicación Audiovisual y Publicidad, Universidad CEU Cardenal Herrera, Valencia, España.

León Santos, M. (2008).Propuesta deun modelo demedición delagestión del conocimiento para organizaciones de Información. Tesis de Doctorado, Universidad de La Habana, Cuba.

Lehtonen, M. (2009). Nonaka冈s knowledge creation theory revisited: A Semiotic. Analysis of Communicating Knowledge in a Geographically Dispersed Team. Available on: https://www.academia.edu/197587/Nonaka_s_knowledge_creation_ theory_revisited_a_semiotic_analysis_of_communicating_knowledge_in_a_ geographically_dispersed_team

Leite, F.C.,(2006). Gestão do Conhecimento Científico: Proposta de um modelo conceitual. Dissertação de Mestrado Programa de Pós-Graduação em Ciência da Informação, Universidade de Brasília, Brasil.

Leite,F.C.(2007).Comunicação científica egestão do conhecimento: enlaces conceituais para fundamentação da gestão do conhecimento científico no contexto de universidades. Transinformação, 19(2), 139-151.

Liyanage, C., Ballal, T., Elhag, T. \& Li, Q. (2009). Knowledge communication and translation- A knowledge transfer model. Journal of Knowledge Management, 13(3), 118-131.

Lopera Londoño, M. E. \& Quiroz Gil, N. L. (2013). Caracterización de un modelo de gestión del conocimiento aplicable a las funciones universitarias de investigación y extensión: caso Universidad CES - Universidad del Rosario. Tesis de Maestría, Medellín, Colombia. 
López Jiménez, D. F. (2006). El conocimiento y la comunicación: dos pilares fundamentales de la organización de la sociedad de la información. Palabra Clave, 9(2), 91-109. Recuperado de: http://www.redalyc.org/articulo. oa? $\mathrm{id}=64900209$

Martín Barbero, J. (2009). La Universidad vive hoy en el mundo una relación esquizofrénica con la sociedad. Conferencia magistral. XIII Encuentro de FELAFACS, La Habana, Cuba. Recuperado de: http://www.cubadebate.cu/

Martín Martín, F. (2011). El plan estratégico de comunicación como nuevo modelo de investigación científica universitaria. Correspondencias \& Análisis, 1, 102-114.

Meza, P. (2013). La comunicación del conocimiento en las secciones de Tesis de lingüística: determinación de la variación entre grados académicos. Tesis de Doctorado, Pontificia Universidad Católica de Valparaíso, Chile.

Meza, P. (2015). La comunicación del conocimiento en el género Tesis de Lingüística: Comparación entre grados académicos. En G. Parodi \& G. Burdiles (eds.), Leer yescribir en contextos académicos y profesionales (pp. 67-112). Santiago de Chile: Planeta.

Molina, J. L. \& Marsal Serra, M. (2002). Lagestión del conocimiento en las organizaciones. Buenos Aires: Libros en red.

Mohr, S.M. (2007). The Role of Communication in Knowledge Management and Knowledge Exchange in Organizations, Unpublished $\mathrm{PhD}$ dissertation, Graduate Program in Communication, Information and Library Studies, University of New Jersey United State.

Munir \& Rohendi, D. (2012). Development Model for Knowledge Management System (KMS) to Improve University's Performance. Case Studies in Indonesia University of Education. IJCSI International Journal of Computer Science Issues, 9(1), 1-6.

Nonaka, I. \& Takeuchi, H. (1995). The knowledge-creating company. New York: Oxford University Press.

Tingoy, Ö. \& Kurt, Ö. (2009). Communication in knowledge management practices: A survey from Turkey. Problems and Perspectives in Management, 7(2), 46-52.

Passoni, L. I. \& Zanfrillo, A. (2003). Un Modelo de gestión del conocimiento en los Departamentos Académicos. Centro de Investigaciones dela Gestión Universitaria. Facultad de Ciencias Económicas y Sociales. Argentina.

Pérez-Montoro Gutiérrez, M. (2008). Gestión del conocimiento en las organizaciones. Fundamentos, metodología y praxis. España: Trea.

Pérez Rodríguez, Y. \& Castañeda Pérez, M. (enero-abril, 2009). Redes de conocimiento. Ciencias de la Información, 40(1), 3-20. 
Pérez Sánchez, E. O., Ramírez Quintero, G. \& Gallego Mosquera, N. I. (2011). Modelo de gestión del conocimiento para el Instituto Tecnológico Metropolitano-ITM. Tesis de Maestría en Administración, Facultad de Ciencias Económicasy Administrativas, Universidad de Medellín, Colombia.

Ponjuán Dante, G. (2006). Introducción a la gestión del conocimiento. La Habana: Félix Varela.

Probst, G., Raub, S. \& Romhardt, K. (2001).Administre el conocimiento. México: Pearson Educación.

Puertas Hidalgo, R., Cadme, E. \& Álvarez Nobell, A. (2015). Gestión estratégica de la comunicación digital en la empresa ecuatoriana. Perspectiva comparada con la realidad europea. Revista Internacional de Relaciones Públicas, 9(5), 5-26.

Reina Estévez, J., Fernández Castillo, I. \& Noguer Jiménez, A. (2012). El uso de las redes sociales en las universidades andaluzas: El Caso de Facebook y Twitter. Revista Internacional de Relaciones Públicas, 4(2), 123-144. Recuperado de: es/index.php/ revrrpp/article/view/128

Rizo García, M. (2014). El papel de las teorías de la comunicación en la construcción del campo académico de la comunicación. Reflexiones desde la historia, la epistemología y la pedagogía. Correspondencias \& Análisis, 4, 239-258.

Rodríguez Andino, M. et al. (2009). La gestión del conocimiento, factor estratégico para el desarrollo. Gestión en el Tercer Milenio, Rev. de Investigación de la Facultad de Ciencias Administrativas, UNMSM, 12(23), 7-14.

Rojas Vera, L. \& Arapé Copello, E. (1999). La Visión y la Comunicación en la Gerencia. Revista Opción, 28, 29-58.

Romero Frías, E. \& Robinson García, N. (2017). Laboratorios sociales en universidades: Innovación e impacto en Medialab UGR. Comunicar, XXV(51), 29-38.

SaladrigasMedina,H.(2018).LagestióndelaComunicaciónOrganizacional/Institucional. Recuperado de: https://www.researchgate.net /publication /330158433

Scheinsohn, D. (1993). Comunicación estratégica. Management y fundamentos de la imagen corporativa. Córdoba: Ediciones Macchi.

Straub, D. \& Karahanna, E. (1998). Knowledge Worker Communications and Recipient Availability: Toward a Task Closure Explanation of Media Choice. Organization Science, $9(2), 160-175$.

Szulanski, G. (1996). Exploring internal stickiness: Impediments to the transfer of best practice within the firm. Strategic Management Journal, 17, 29-46.

Timm, P. (1986). Managerial Communication: a finger on the pulse. Prentice Hall: Englewood Cliffs. 
Treem, J. W. (2012). Communicating expertise: Knowledge performances in professional-service firms. Communication Monographs 79(1), 23-47.

Valentim, M.L.(2005). Construção de conhecimento científico. En Métodos qualitativos de pesquisa em Ciência da Informação. (s.p.). São Paulo: Polis.

Van Buren, M.E. (2000). Midiendolagestión del conocimiento. Training ઐ Development Digest, 22, 70-77.

Vargas, L. (2003). Gestión Comunicacional e Identidad Corporativa de la Organización Universitaria. Tesis de Maestría, Ciencias de la Comunicación, Facultad de Humanidades y Educación, Universidad de Zulia, Venezuela.

Wiig, K.M. (2002). New Generation Knowledge Management: What May we Expect? Nowledge Research Institute, Inc. Available on: http://www.krii.com/downloads/ new_gen_km.pdf

* Contribución: El trabajo fue organizado de manera conjunta y corresponden porcentajes iguales.

${ }^{*}$ Nota: El editor Paul Capriotti y el Comité Editorial de la revista aprobaron la publicación del artículo. 


\section{IDENTIFICACIÓN DE LES AUTORE......}

Viena Medina González. Máster (2019) en Dirección Estratégica e Innovación en Comunicación por la Universidad de Málaga, España. Máster (2018) en Bibliotecología y Ciencias de la Información de la Universidad de La Habana, Cuba. Doctoranda en el Programa de Doctorado Interuniversitario de Comunicación de las Universidades de Málaga, Cádiz, Sevilla y Huelva (España); en la línea de investigación Publicidad y Relaciones Públicas. Licenciada (2011) en Bibliotecología y Ciencias de la Información dela Universidad de La Habana, Cuba. Ha sido Jefa de Centro de Información y Biblioteca y docente en la Universidad de La Habana, en el área de la gestión de información y el conocimiento. Coordinadora y especialista en proyectos institucionales, en temas de información, comunicación, gestión del conocimiento y desarrollo de competencias. Autora de varios artículos como: "Gestión del conocimiento y capital social: su relación en contextos universitarios" (2017, Revista Cubana de Información en Ciencias de la Salud); "Modernizando la administración pública: la dimensión infocomunicativa del cambio de mentalidad" -Proyecto en coautoría ganador del Premio Academia de Ciencias de Cuba- (2018, Anales de la Academia de Ciencias de Cuba); "Propuesta de acciones orientada a la gestión del conocimiento y de la información en el desarrollo local” -coautoría con Yasneidy Del Río López, Gretel Lobelle Fernández, Amanda Talía Castillo Carratalá- (2019, Revista Cubana de Información en Ciencias de la Salud).

Ana Almansa Martínez. Doctora en Comunicación por la Universidad de Málaga, España. Profesora Titular de Universidad, Facultad de Ciencias de la Comunicación, Universidad de Málaga. Autora de más de cincuenta artículos en revistas científicas y algunos libros. Entre ellos: Assessorías de Comunicaçao (2010. Difusão Editora); Del gabinete deprensa al gabinete de comunicación. La dirección de comunicación en la actualidad (2011, Comunicación Social Ediciones y Publicaciones); Teoría, Estructuray Funcionamiento de los Gabinetes de Comunicación (2004, SPUMA); Nuevos medios en Comunicación Política -coautoría con Antonio Castillo Esparcia- (2015, Asociación de la Prensa de Huelva). Investigadora principal en el proyecto de investigación Lobby y Comunicaciónen España. Análisis de las estrategias de comunicación (Programa Nacional de I+D, Ministerio de Ciencia e Innovación, España) y del proyecto La Comunicación de los bienes patrimoniales para el desarrollo económico y social de Andalucía (Centro de Estudios Andaluces, España). Editora de la Revista Internacional de Relaciones Públicas. Coordinadora del Máster Oficial "Dirección Estratégica e Innovación en Comunicación". Secretaria dela Comisión Académica en Málaga del "Doctorado Interuniversitario en Comunicación”.

Antonio Castillo Esparcia. Doctor en Comunicación por la Universitat Autónoma de Barcelona (UAB), España. Catedrático dela Universidad de Málaga. Actualmente, Director del Grupo de Investigación Las Relaciones Públicas en la pequeña y mediana empresa. Enla actualidad, Presidente dela Asociación de Investigadores en Relaciones Públicas (AIRP). Director del Proyecto de Investigación Lobby y Comunicación, financiado por el Programa Estatal de I+D+i, Ministerio de Ciencia e Innovación, España (CSO2016-79357-R). Director del Departamento de Comunicación Audiovisualy Publicidad y Coordinador del Máster "Dirección Estratégica e Innovación en Comunicación”, de la Universidad de Málaga. Es evaluador dela Agencia Nacional de Evaluación dela Calidad y Acreditación(ANECA), la Agència per a la Qualitat del Sistema Universitari de Catalunya (AQU), la Agencia de Calidad del Sistema Universitario Vasco (UNIBASQ) y la Agencia Estatal de Investigación. Autor de más de 170 publicaciones, entre artículos en revistas especializadas, libros y capítulos de libros en editoriales nacionales y extranjeras. Entre ellos pueden mencionarse: Lobby y Comunicación (2011, Comunicación Social); Los Think Tanks en España -coautoría con Sergio Guerra- (2019, Tirant lo Blanch); Introducción a las Relaciones Públicas (2010, Instituto de Investigación en Relaciones Públicas).

\section{REGISTRO BIBLIOGRAFICO}

Medina González, V., Almansa Martínez, A. \& Castillo Esparcia, A. (enero-junio, 2020). Comunicación y gestión del conocimiento en las universidades. Divulgar en entornos digitales. InMediaciones de la Comunicación, 15(1), 41-66. 